\title{
Multi-Scale Modelling to Relate Beryllium Surface Temperature, Deuterium Concentration and Erosion in Fusion Reactor Environment
}

\section{Safi, Elnaz}

2017-03-27

Safi , E , Valles , G , Lasa Esquisabel , A \& Nordlund, K H 2017 , ' Multi-Scale Modelling to Relate Beryllium Surface Temperature, Deuterium Concentration and Erosion in Fusion

Reactor Environment ' , Journal of Physics. D, Applied Physics , vol. 50 , no. 20 , 204003 . https://doi.org/10.1088/13

http://hdl.handle.net/10138/308773

https://doi.org/10.1088/1361-6463/aa6967

cc_by_nc_nd

acceptedVersion

Downloaded from Helda, University of Helsinki institutional repository.

This is an electronic reprint of the original article.

This reprint may differ from the original in pagination and typographic detail.

Please cite the original version. 
Preprint of paper published as ournal of Physics. D, Applied Physics. 50, 22 (2017) 225302.

\title{
Multi-Scale Modelling to Relate Beryllium Surface Temperature, Deuterium Concentration and Erosion in Fusion Reactor Environment
}

\author{
E. Safi ${ }^{\mathrm{a}}$, G. Valles ${ }^{\mathrm{b}}$, A. $\operatorname{Lasa}^{\mathrm{c}}$, K. Nordlund ${ }^{\mathrm{a}}$ \\ ${ }^{a}$ University of Helsinki, PO Box 43, 00014 University of Helsinki, Finland \\ ${ }^{b}$ Instituto de Fusión Nuclear, Universidad Politécnica de Madrid, Madrid, Spain \\ cOak Ridge National Laboratory, Oak Ridge, TN 37920 USA
}

* Corresponding author.
E-mail address: Elnaz.safi@helsinki.fi (E. Safi).

\begin{abstract}
Beryllium (Be) has been chosen as plasma facing material for the main wall of ITER, the next generation fusion reactor. Identifying the key parameters that determine Be erosion under reactor relevant conditions is vital to predict the ITER plasma facing component lifetime and viability. To date, a certain prediction of Be erosion, focusing on the effect of two of such parameters, surface temperature and D surface content, has not been achieved. In this work, we develop the first multiscale KMC-MD modeling approach for Be to provide a more accurate database for its erosion, as well as investigating parameters that affect erosion. First, we calculate the complex relationship between surface temperature and $\mathrm{D}$ concentration precisely by simulating the time evolution of the system using an object kinetic monte carlo (OKMC) technique. These simulations provide D surface concentration profile for any surface temperature and incoming D energy. We then describe how this profile can be implemented as a starting configuration in molecular dynamics (MD) simulations. We finally use MD simulations to investigate the effect of temperature (300-800K) and impact energy $(10-200 \mathrm{eV})$ on the erosion of Be due to D plasma irradiations. The results reveals a strong dependency of the D surface content on temperature. Increasing the surface temperature leads to a lower $\mathrm{D}$ concentration at the surface, because of the tendency of $\mathrm{D}$ atoms to avoid being accommodated in a vacancy, and de-trapping from impurity sites and diffuse fast toward bulk. At the next step, total and molecular Be erosion yields due to D irradiations are analyzed using MD simulations. The results show a strong dependency of erosion yields on surface temperature and incoming ion energy. The total Be erosion yield increases with temperature for impact energies up to $100 \mathrm{eV}$. However, increasing temperature and impact energy results in a lower fraction of Be atoms being sputtered as $\mathrm{BeD}$ molecules due to the lower $\mathrm{D}$ surface concentrations at higher
\end{abstract}


temperatures. These findings correlate well with different experiments performed at JET and PISCES-B devices.

\section{Introduction}

With increasing demand of energy in the $21_{\text {st }}$ century, replacing scarce fossil fuels with new energy resources is inevitable. Nuclear fusion power may offer practical, power plant scale energy production with an unlimited fuel supply. During the last decades, a wide range of studies have been carried out to investigate fusion performance and fusion reactor designs. ITER [1] will be the first experimental tokamak-like nuclear fusion reactor to produce net energy, based on deuterium (D)tritium (T) plasmas [2]. Due to the ITER design and operation requirements, extreme conditions are expected for the plasma facing components (PFCs) such as thermal loads, temperature and particle fluxes. Therefore, selecting appropriate materials for different components of the device is critical and highly demanding.

The ITER blanket system, which is designed to slow down high-energy neutrons coming from plasma and to extract their heat energy, is one of such components that directly face the confined plasma [3]. Beryllium (Be) has been chosen as first wall material of ITER blanket, due to its low fuel retention, low plasma contamination and its oxygen gattering properties [2,4]. As a result of non-perfect plasma confinement, Be will be exposed to D plasma, leading to erosion of the wall material. Erosion of PFCs can become a show-stopper by limiting their lifetime [5]. Therefore, characterizing the sputtering of Be (by D) and understanding the underlying mechanism is essential toward predicting, and ultimately controlling the adverse effects of plasma-surface interactions (PSI).

A few experiments have been dedicated to study these PSIs by exposing Be targets to D plasmas, for instance, in the JET tokamak [6] and the PISCES-B linear plasma facility [7-9], as well as using ion beams [10]. The experiments show a complex erosion behavior; consisting of e.g. release of BeD molecules [8], change of D surface content, as well as temperature dependent erosion yield $[5,9,10]$. Such uncertainties hinder reliable prediction of Be erosion in future fusion reactors. In addition to experiments, computer simulation of the Be-D system has also contributed to a better characterizing erosion and gaining insight of the underlying mechanism. For instance, Be erosion and redeposition, along with emission profiles and chemical erosion of $\mathrm{BeD}$ molecules, have been 
accurately modeled by ERO calculations [11] across several devices. Erosion of pure Be and Bebased materials exposed to $\mathrm{D}$ has also been studied widely at the atomic scale, mainly by molecular dynamics (MD), initially focused on the effect of impact energy and flux, but recently also including surface temperature and D surface concentration [9,12-16].

However, a complete description of the latter effects had not been achieved to this date. Our previous study, MD simulation of cumulative D impacts on Be surfaces, showed a complex outcome for molecular erosion [16]. Due to very different D surface content at different surface temperatures, larger Be-D molecules were also emitted when the D concentration increased in the topmost layer. These results were unphysical for reactor-like conditions, caused by MD being limited to short time scales, and consequently high irradiation fluxes [15]. However, MD is a valuable tool to study low-energy irradiation effects in materials and quantifying erosion yields in order to gain insight in the underlying processes.

Therefore, the present study was designed to investigate erosion of equilibrated Be surfaces, in an kinetic Monte Carlo (KMC)-MD multi-scale approach. The complex relationship between surface temperature and D surface content has been appropriately calculated by longer time-scale approach, KMC technique. The KMC outcome provides initial substrate structures for MD closer to experimental condition, which allows gaining insight into effect of surface temperature and irradiation energy on Be erosion. Further, this approach is able to offer a more precise database of erosion yields to the large scale impurity transport codes such as ERO [11].

\section{Method}

\section{$2.1 \mathrm{KMC}$}

\subsubsection{Overview}

The kinetic Monte Carlo (KMC) method is a modeling technique aimed to simulate the time evolution of a system by randomly sampling the different processes that may happen in the system, according to their probabilities, i.e., rates [17]. When the modeled processes are enforced for objects such as impurities or defects, the method is known as Object Kinetic Monte Carlo (OKMC).

OKMC is one of the preferred techniques to study defect evolution in solid materials [18-20]. The 
model assumes that there are several different states in a system and the transitions between these states are known and independent of time. These transitions are the input parameters of the algorithm, and the dynamic evolution of the system out of equilibrium is followed by OKMC [2123]. Since OKMC only takes into account defects (self-interstitial atoms, vacancies, impurities and their clusters) but not the lattice atoms themselves, simulation boxes of the order of few $\mu \mathrm{m}$ and large time scales of the order of hours (even days) can be simulated. Different OKMC algorithms exist, depending on the modelled processes, objects, probabilities and interactions. One of such models designed to study long term evolution of damage in irradiated solids, for instance in fusion reactor materials, is called MMonCa [24].

In MMonCa, the transition rates (migration and dissociation events) are modelled as Arrhenius laws with an activation barrier and a prefactor. In this simulator, defect evolution in solids are followed by three modules: 1) objects or defects and all their possible transition rates and interactions 2) computing time evolution by a rate manager 3) creating spatial transitions and defect interactions by a space manager [24].

In MMonCa simulator the objects are defined as defects that introduce undesired effects on materials applications (here in ITER fusion reactor). MMonCa needs two sorts of input data: (i) an activation energy and a prefactor for each transition that might happen, such as migration or dissociation of defects and (ii) the initial positions of the defects present at the beginning of the simulation. Further details regarding function implementation, as well as more thorough description of objects diffusion and reactions, can be found in ref. [24].

Previously, the MMonCa has been successfully applied to study low energy helium irradiation on tungsten in a wide range of temperatures to determine tungsten fuzz growth [25]. Here, we used MMonCa to investigate the effect of surface temperature on D depth profile in Be.

\subsubsection{Parametrization}

OKMC simulations were performed using MMonCa code to study the relationship between Be surface temperature and D diffusion and depth profile in Be. Single D atoms, carbon (C), vacancy (V) and a D atom trapped in a vacancy (DV) are considered as objects, which can migrate, dissociate from or pile into clusters in the system. The barrier for migration and formation of all mobile objects are parametrized in table 1 . These values are obtained from literatures mostly 
obtained with density functional theory (DFT) calculations [31]. Since Be has a close-packed atomic structure, the concentration of intrinsic interstitial defects is smaller and defects are completely dominated by vacancies [26]. Therefore, Be interstitial defects are not taken into account during this study. Detail description about mobile objects parameterization and validation considered in this study, are explained below.

In fusion reactors, irradiation damage or thermal excitations will generate vacancies in the Be cell. Hence, in this study various vacancy concentrations $\left(\mathrm{C}_{\mathrm{v}}\right)(0,1,5,10$ and $20 \%)$ were introduced in Be cell, bearing in mind that formation of divacancies is energetically unfavorable due to their negative binding energy [33].

Another dynamic mobile particle in our system is D. Hydrogen isotopes are known to be successfully trapped in Be [29]. D tends to be trapped in a V and the V sites are filled first before the interstitial sites [28]. Eventually, V traps become saturated and untrapped D atoms diffuse as an interstitial either deeper in the material or toward the surface [34]. However, ab initio studies shows that two D atoms trapped in a vacancy cannot form a molecule, and dissociate into two separate D atoms $[29,32,33]$.

$\mathrm{D}$ is greatly attracted by vacancies in Be, therefore a single $\mathrm{D}$ prefers to remain in a $\mathrm{V}$ and form a mobile D-V particle, due to D having a lower detrapping energy than trapping energy $[28,32]$. When a D occupies a V site still plenty of space, remains in V to accommodate more D [29]. DFT calculations report that up to five separated D atoms can be held in a monovacancy site in Be solid, accordingly, all the interactions of D with DV are introduced in our simulations.

$$
D_{n} V+D \rightarrow D_{n+1} V ; n=1-4
$$

Nevertheless, $D_{n+1} V$ particles are considered immobile in Be [28,32].

In order to create more trapping sites and make the simulation more realistic, $1 \% \mathrm{C}$ impurities as mobile particles, were also introduced to the system. These impurities were accommodated in Be interstitial sites and the migration barrier for extrinsic interstitial $\mathrm{C}$ in $\mathrm{Be}$ was calculated in this work, using the nudged elastic band (NEB) technique $[35,36]$. The simulation was carried out in a way that $\mathrm{C}$ atoms are able to trap up to four $\mathrm{D}$ atoms and become immobile. The interactions of $\mathrm{D}$ atoms with $\mathrm{C}$ were introduced to the system. 


$$
D_{n} C+D \rightarrow D_{n+1} C ; n=1-3
$$

\subsubsection{Simulation set-up}

An elongated box of Be with dimensions $X=10 \mathrm{~nm}, \mathrm{Y}=10 \mathrm{~nm}$ and $\mathrm{Z}=100 \mathrm{~nm}$ was created to perform simulations. The depth of the simulation box $(100 \mathrm{~nm})$ was considered long enough to allow the simulation of diffusion and migration of $\mathrm{D}$ ions in Be. The free surface was considered at top Z surface, considered as a desorption surface. Furthermore, atoms reaching the bottom of the cell in $\mathrm{Z}$ direction were considered to migrate deeper into the bulk and hence ignored. Moreover, periodic boundary conditions (PBCs) were applied in $\mathrm{X}$ and $\mathrm{Y}$ directions in order to model an infinite surface.

The initial positions of implanted D ions were calculated with the SRIM code [37], by irradiating Be with $\mathrm{D}$ ions at $50 \mathrm{eV}$. The ion flux was $10^{24} \mathrm{~m}^{-2} \mathrm{~s}^{-1}$, taking into account the reflection yield provided by SRIM. Different vacancy concentrations and 1\% carbon impurities were also inserted in the simulation cell. Finally, D irradiation simulations in Be, at impacting energy of $50 \mathrm{eV}$, and the time evolution of objects were performed at six different temperatures (300, 400, 500, 600, 700 and $800 \mathrm{~K})$, with the main aim of calculating the D surface content in Be. The simulation times were up to 1.2 seconds.

In our OKMC simulations many aspects of D migrations and defect diffusions in ITER first-wall relevant conditions, were examined. Eventually, D distribution profile at different temperatures was calculated from OKMC to set up accurate substrate structures in MD. Due to MD time scale limitations, diffusion evolution processes are not accessible by MD, therefore, OKMC will assist MD to reproduce the simulation conditions closer to experimental conditions.

\subsection{MD}

\subsubsection{Overview}

Molecular dynamics (MD) is an atomistic scale computational method suitable for studying atomic bond breaking and formation when appropriate reactive interatomic potentials are used. Thus, it is a suitable technique to investigate the erosion mechanism of chemically active systems under highly 
controlled conditions, such as systematically varying desired parameters.

Here, D irradiation of Be surfaces was simulated with the MD code PARCAS [38]. The evolution of the system is explained here by the forces between the atoms, defined by interatomic potentials. To calculate the interatomic forces during our simulations, a many-body bond-order classical potential [39] was used. This potential is classical, although its parameterization is derived from quantum mechanical calculations.

\subsubsection{Simulation set-up}

The MD simulations were carried out in two main steps: first mixed Be-D surfaces were prepared, and then the D irradiation was performed.

The cells were initially created by relaxing a hexagonal closed packed structure (HCP) at the desired temperatures, including opening of the perfectly flat $\left(\begin{array}{llll}0 & 0 & 0 & 1\end{array}\right)$ surface normal in the $\mathrm{Z}$ direction. The cells originally consisted of 6048 Be atoms in a box of dimensions $\mathrm{X}=2 \mathrm{~nm}, \mathrm{Y}=2.4$ $\mathrm{nm}$ and $\mathrm{Z}=10.1 \mathrm{~nm}$. After the initial relaxation, The Be substrate structures were modified to accommodate $\mathrm{D}$ and vacancy profiles given by the OKMC runs for each temperature. The procedure was handled with special care by first creating vacancies in the relaxed initial cells and then re-relaxing new cells. Next, D atoms were inserted according to D surface content profiles given by OKMC, taking into account the number of $\mathrm{D}$ accumulated in a $\mathrm{V}$ at different temperatures. In order to keep the created cells close to the equilibrium conditions, the Be cells were relaxed after each D implantation for 10 ps.

After creating the new equilibrium Be cells, these Be surfaces were irradiated with 4000 noncumulative D impacts varying the impact energies (10, 30, 50, 100, 150 and $200 \mathrm{eV}$ ) and surface temperatures (300, 400, 500, 600, 700 and $800 \mathrm{~K}$ ). All the impacts were performed normal to the surface. All irradiations were carried out non-cumulatively in order to keep the substrate equilibrium and create more controlled conditions by keeping D surface contents constants over substrate morphologies. Therefore, only the impact point was varied in each event, by randomly shifting the cell in $\mathrm{X}$ and $\mathrm{Y}$ directions. 
Further details regarding the methodology, use of PBCs, electronic stopping, pressure and temperature control and treatment of sputtered and backscattered particles, can be found in ref. [40].

\section{Results}

\subsection{OKMC}

Figure 1 illustrates equilibrium D distribution in Be for different vacancy concentrations for two different temperatures. The number of $\mathrm{D}$ atoms in a given depth range is normalized to the density of Be atoms in the bulk. As can be seen from both graphs, D atomic density can reach high values, especially near the surface. At 400K surface temperature, D atoms accumulate only in the first few surface layers, while in layers deeper than $6 \mathrm{~nm}$, there is almost zero D concentration. With increasing temperature, $\mathrm{D}$ atoms distribute deeper and more evenly in the cell, as revealed in the right-hand graph of figure 1. Moreover, a linear dependency of $\mathrm{D}$ distribution on vacancy concentration is reported. As mentioned earlier vacancies are more favorable defects to accommodate D atoms rather than interstitial sites in Be, therefore, higher vacancy concentration provides more traps for $\mathrm{D}$ atoms to accommodate and hence higher $\mathrm{D}$ concentrations. It should be noted that $\mathrm{D}$ depth profiles are varying weakly for 0-10\% vacancy concentrations.

The OKMC results indicate that at higher temperatures, D atoms distribute more evenly and deeper in the cell, and therefore D surface contents decrease by increasing temperature. Figure 2 illustrates the final D profiles at 5\% vacancy concentration (reasonable for mixed crystalline, and co- and redeposited layers) to set up accurate substrate structures in MD at different temperatures.

The quantities displayed in table 2 show the number of $\mathrm{D}$ atoms trapped in a vacancy at the different simulation cases studied here. A strong temperature dependency of D concentration on the number of vacancies is observed during the simulations. At the early stages of our simulation, all vacancies were filled with up to $5 \mathrm{D}$ atoms and all other incoming $\mathrm{D}$ atoms diffused in the cell as interstitials. $\mathrm{D}$ atoms tend to accommodate in a vacant site rather than becoming interstitials. As the simulation goes on, at lower temperatures $\mathrm{D}$ atoms remain in vacancies and form immobile $\mathrm{D}_{5} \mathrm{~V}$ defects. At higher temperatures, $\mathrm{D}$ atoms are de-trapped from vacancies regularly because of their lower binding energy and diffuse fast toward bulk as an interstitial. 


\subsection{MD}

This study is carried out to focus on the interaction between the prepared in equilibrium amorphous Be surfaces and irradiating D ions. Therefore, there is no interest in further evolving the surfaces and hence the non-cumulative simulations were performed. The total Be erosion yield at different surface temperature and irradiation energy is represented in figure 3. The results clearly show how different D surface contents of Be cells at different temperatures affects the Be erosion behavior. With increasing surface temperature and hence reduced D surface concentration, the erosion yield of Be increases within the error bar, for ion energies greater than $10 \mathrm{eV}$. At lower surface temperatures where D concentrations are higher (there are more D neighbors to surface Be atoms), the probability of hitting a Be atom with incoming ion is lower than higher surface temperature, which leads to lower total Be erosion yield.

The ion energy dependency of the total Be erosion yield is significant (Fig. 3). At $10 \mathrm{eV}$ impact energy, it is highly unfavorable for Be atoms to receive enough energy to erode from the surface at normal incidence, therefore, there is almost zero sputtering yield at this impact energy. With increasing impact energy, the total Be erosion yield increases with temperature, where it peaks at $100 \mathrm{eV}$. For energies higher than $100 \mathrm{eV}$ a reduction in total yield is observed, which is due to larger penetration depth of D ions at higher energies, which lowers the surface interactions.

Figure 4 shows a comparison of modelled and measured total Be sputtering yield at 300K surface temperature as a function of incident ion energy. The experimental yields were measured in PISCES-B facility, using a polycrystalline Be sample [41]. The sputtering yield of Be atoms calculated by Eckstein's theoretical model [42], scaled by a factor of 0.5, is also plotted. The MD data follows a similar trend as the Eckstein data, however, the values are much lower. The difference between MD and experimental yields is considerable specially for impact energies higher than $60 \mathrm{eV}$. It is believed that the reason for this difference is related to D surface content and surface morphology changes, however, the experimental yields still follow the same trend as in MD simulations.

The fraction of Be atoms that are sputtered as $\mathrm{BeD}$ molecules is illustrated in figure 5 . The results show a high surface temperature and impact energy dependency, where increasing both parameters 
lead to a reduction of the $\mathrm{BeD}$ erosion yield. At higher temperatures and hence lower $\mathrm{D}$ concentration, there are less $\mathrm{D}$ atoms than what is initially bound to Be atoms, which lead to lower BeD erosion yield. Moreover, as the impact energy increases, single Be atoms are eroded via physical sputtering rather than swift chemical sputtering [14], lowering the BeD molecular fraction. It should be noted that among the sputtered species there were around $1 \% \mathrm{BeD}_{2}$ molecules of all BeD molecules.

Both in the PISCES-B [41] and in the JET tokamak [5] experiments, BeD molecular erosion has also been observed, supporting the incoming ion and surface temperature dependency reported in the present study, which showed a linear drop in BeD:Be ratio with increasing surface temperature (see figure 6). When measuring a BeD signal from Be tiles in the JET first wall, a clear intensity drop is observed in the 500-700K temperature range [5].

\section{Discussions and conclusions}

Keeping the modelling conditions close to fusion reactor-like conditions is essential to reliably study erosion of PFCs. However, parameters such as D concentration and surface structure in the sample are difficult to determine experimentally, and nor is clear how the D concentration affects Be sputtering. Previously, we studied D irradiation on Be scanning over different parameters (temperature, impact energy and flux) by MD simulations only [16]. The dependence of surface temperature on D accumulation and surface morphology was estimated but due to MD time-scale limitations and high ion flux, the confidence in the output was compromised. Later, we prepared samples with various $\mathrm{D}$ concentration in the top $5 \AA$ of the surface at different surface temperatures (decreasing D concentration with increasing temperature) and performed 3000 non-cumulative D irradiations of each sample scanning over incoming ion energy. The results were inconsistent due to wrong D concentration approximation.

Therefore, a multi-scale approach is taken here instead. Equilibrium D depth distribution in Be are first calculated using the OKMC method. Then, the initial MD cells were created, for the D and vacancy distributions given by OKMC. Finally, sputtering yields are calculated by MD.

Equilibrium D distribution profiles are obtained at different surface temperatures. The OKMC 
simulation results confirms a strong dependency of D surface content on the surface temperature. At lower temperatures, a high concentration of D atoms was accommodated close to the Be surface, with the $\mathrm{D}$ being favorably trapped in vacancies. Increasing the temperature made $\mathrm{D}$ atoms to diffuse fast toward bulk and stay in mobile interstitial sites instead. Note, however, that these OKMC simulations are very sensitive to the DFT data used in the parametrization of trapping and de-trapping processes of $\mathrm{D}$ with vacancy.

Total Be and BeD molecular erosion yields, for varying impact energy and surface temperature, were calculated by MD simulations based on the OKMC depth profile. The results indicate a strong temperature (D surface content) and impact energy dependency. The total Be erosion yield increased with the surface temperature and hence with decreasing D concentration, due to higher probability of hitting a Be atom than D by incoming ion. Moreover, erosion yields increased with ion energy up to $100 \mathrm{eV}$, but reduced for energies larger than $100 \mathrm{eV}$ (in the range studied here) because of higher $\mathrm{D}$ penetration depth. One of the main aim for this work is also to calculate the fraction of Be atoms that are sputtered as BeD molecules. MD simulations, confirmed the reduction of sputtered BeD molecules with increasing temperature and ion energy, as reported by experiments done at PISCES and JET, and also obtained by previous MD simulations with temperatureindependent profiles [14].

\section{Acknowledgement}

This work has been carried out within the framework of the EUROfusion consortium and has received funding from the Euratom research and training program 2014-2018 under grant agreement No. 633053. The views and opinions expressed herein do not necessarily reflect those of the European commission. Grants for computer time from CSC, the IT center science in Espoo, Finland, are gratefully acknowledged. 


\begin{tabular}{|l|l|l|}
\hline & $\mathrm{E}_{\text {migration }}(\mathrm{eV})$ & $\mathrm{E}_{\text {formation }}(\mathrm{eV})$ \\
\hline $\mathrm{V}$ & $0.89^{[26]}$ & $0.96^{[27]}$ \\
\hline $\mathrm{D}$ & $0.6^{[28]}$ & $0.88^{[26]}$ \\
\hline $\mathrm{D}-\mathrm{V}$ & $1^{[26]}$ & $0.85^{[29]}$ \\
\hline $\mathrm{C}$ & $0.76^{[\text {from NEB, [30]] }}$ & 0.4 \\
\hline
\end{tabular}

Table 1. Migration and formation barriers of mobile particles ( $\mathrm{V}=$ vacancy, $\mathrm{D}=$ deuterium, $\mathrm{D}-\mathrm{V}=\mathrm{D}$ trapped in a vacancy and $\mathrm{C}=$ carbon) in the beryllium cell.
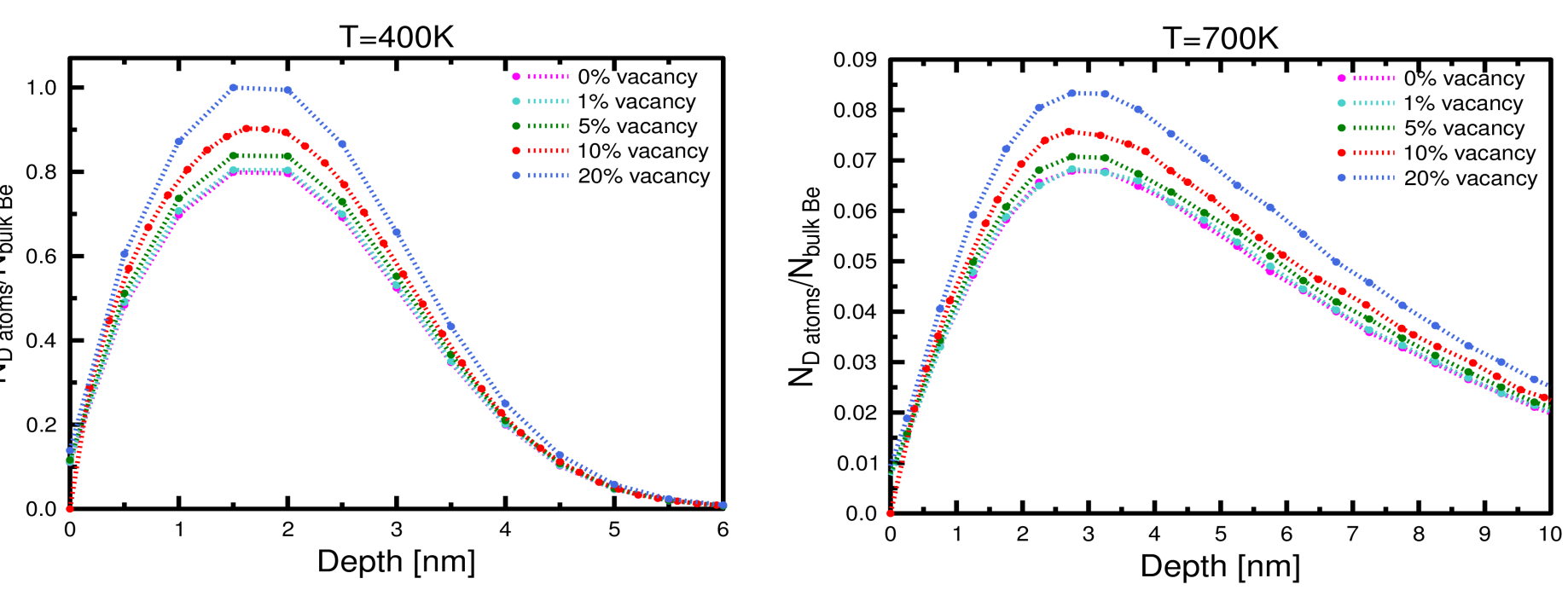

Fig.1 Equilibrium D distribution in Be for different vacancy concentration at $400 \mathrm{~K}$ (left) and 700K (right). The number of $\mathrm{D}$ atoms in different layers are normalized to the number of $\mathrm{Be}$ atoms in bulk. 


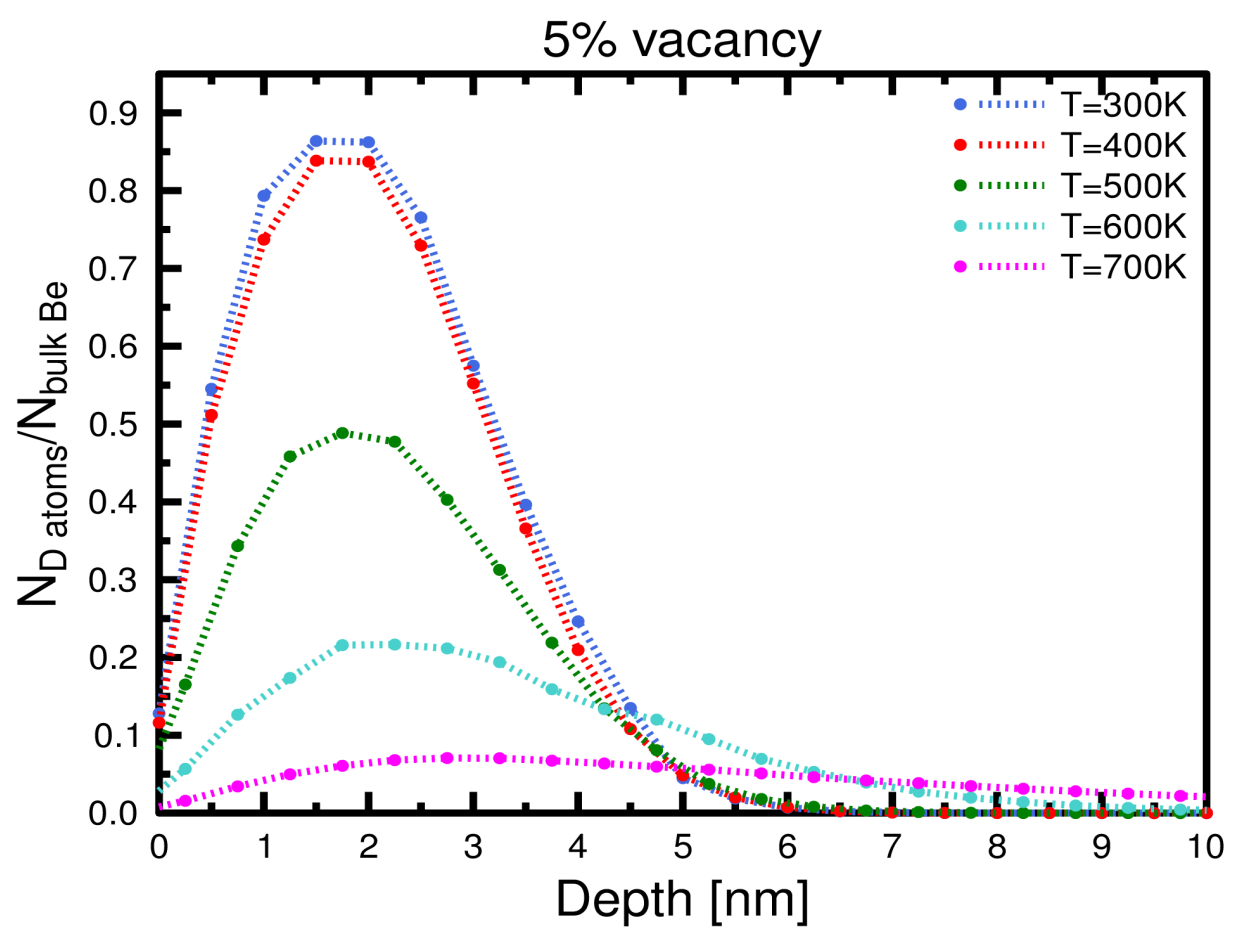

Fig. 2 Final D distribution in Be at 5\% vacancy concentration to set-up MD initial structures at different surface temperatures.

\begin{tabular}{|l|c|c|c|c|c|c|}
\hline & $300 \mathrm{~K}$ & $400 \mathrm{~K}$ & $500 \mathrm{~K}$ & $600 \mathrm{~K}$ & $700 \mathrm{~K}$ & $800 \mathrm{~K}$ \\
\hline $1 \% \mathrm{~V}$ & - & 5 & - & - & 4.11 & - \\
\hline $5 \% \mathrm{~V}$ & - & 5 & - & - & 4.01 & - \\
\hline $10 \% \mathrm{~V}$ & 5 & 4.99 & 4.99 & 4.95 & 3.8 & 1.32 \\
\hline $20 \% \mathrm{~V}$ & 5 & 4.99 & 4.99 & 4.95 & 3.26 & 1.13 \\
\hline
\end{tabular}

Table 2. Number of D atoms trapped in a vacancy site in Be at different surface temperatures and vacancy concentrations. 


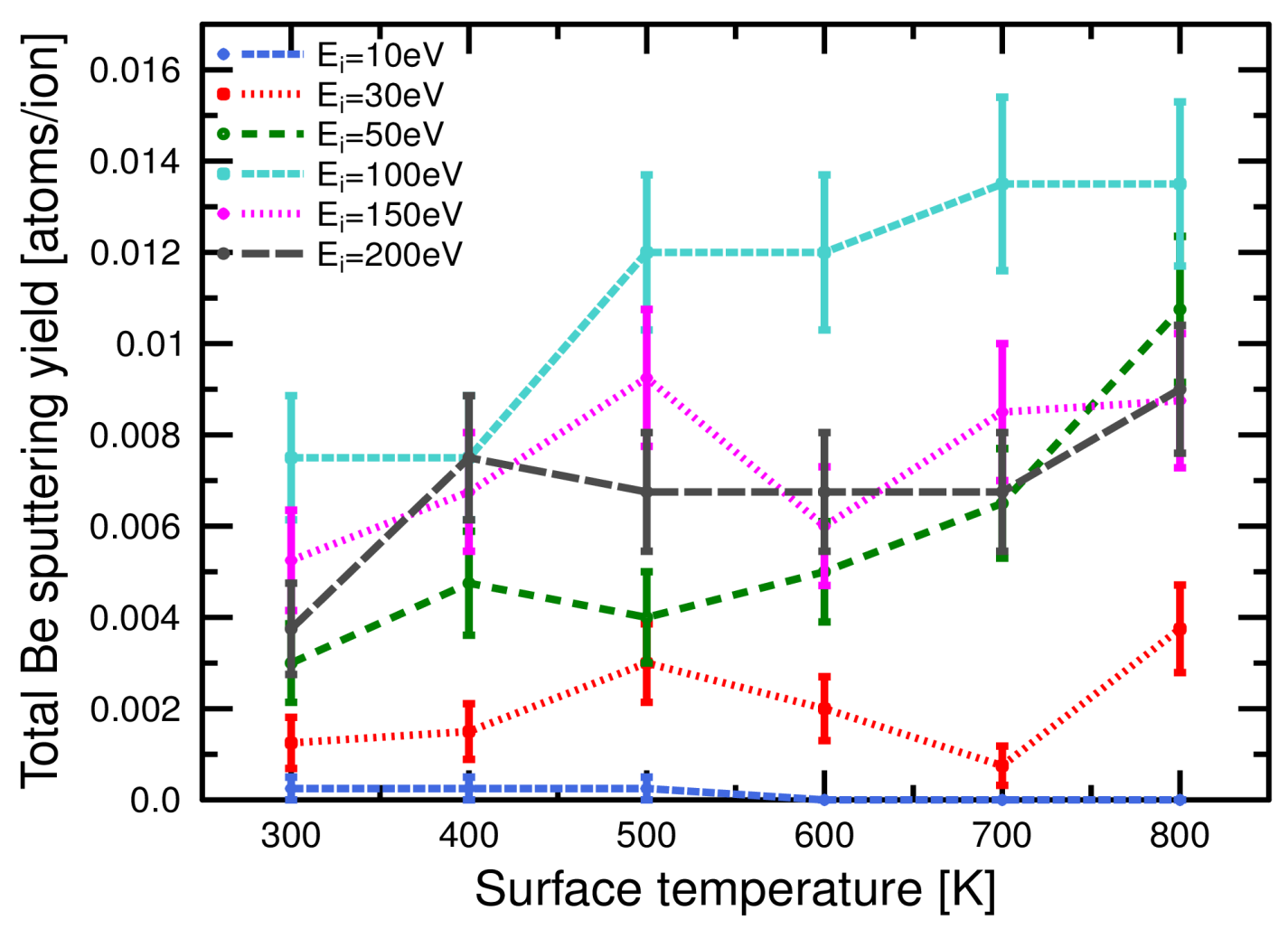

Fig. 3 Total Be sputtering yield by D irradiation as a function of temperature for different impact energies. 


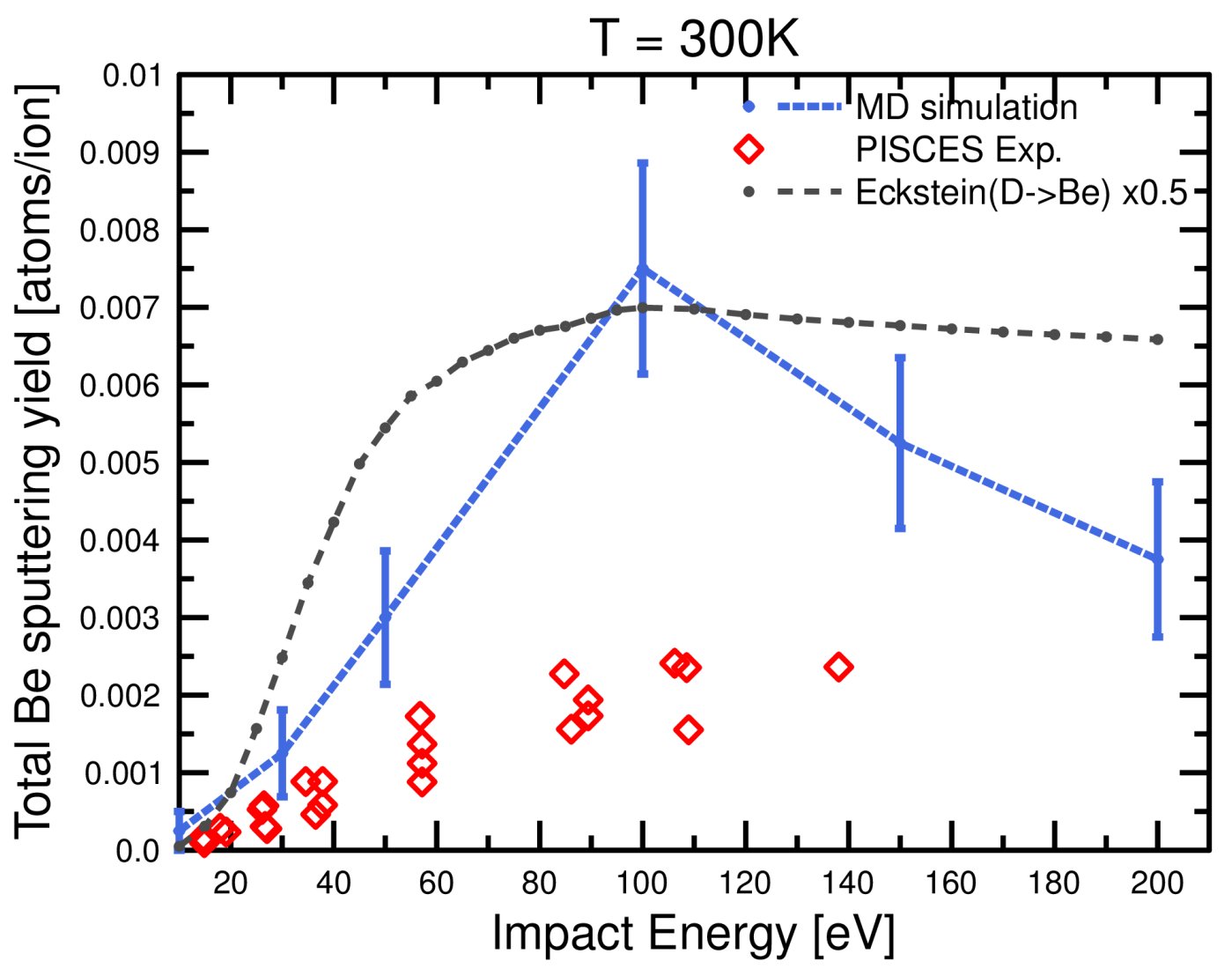

Fig. 4 Comparison of modelled (by MD) and measured (by PISCES-B) total Be sputtering yield by $\mathrm{D}$ irradiations as a function of impact energies at $300 \mathrm{~K}$ surface temperature. The Eckstein theoretical curve is also plotted by a factor of 0.5 . 


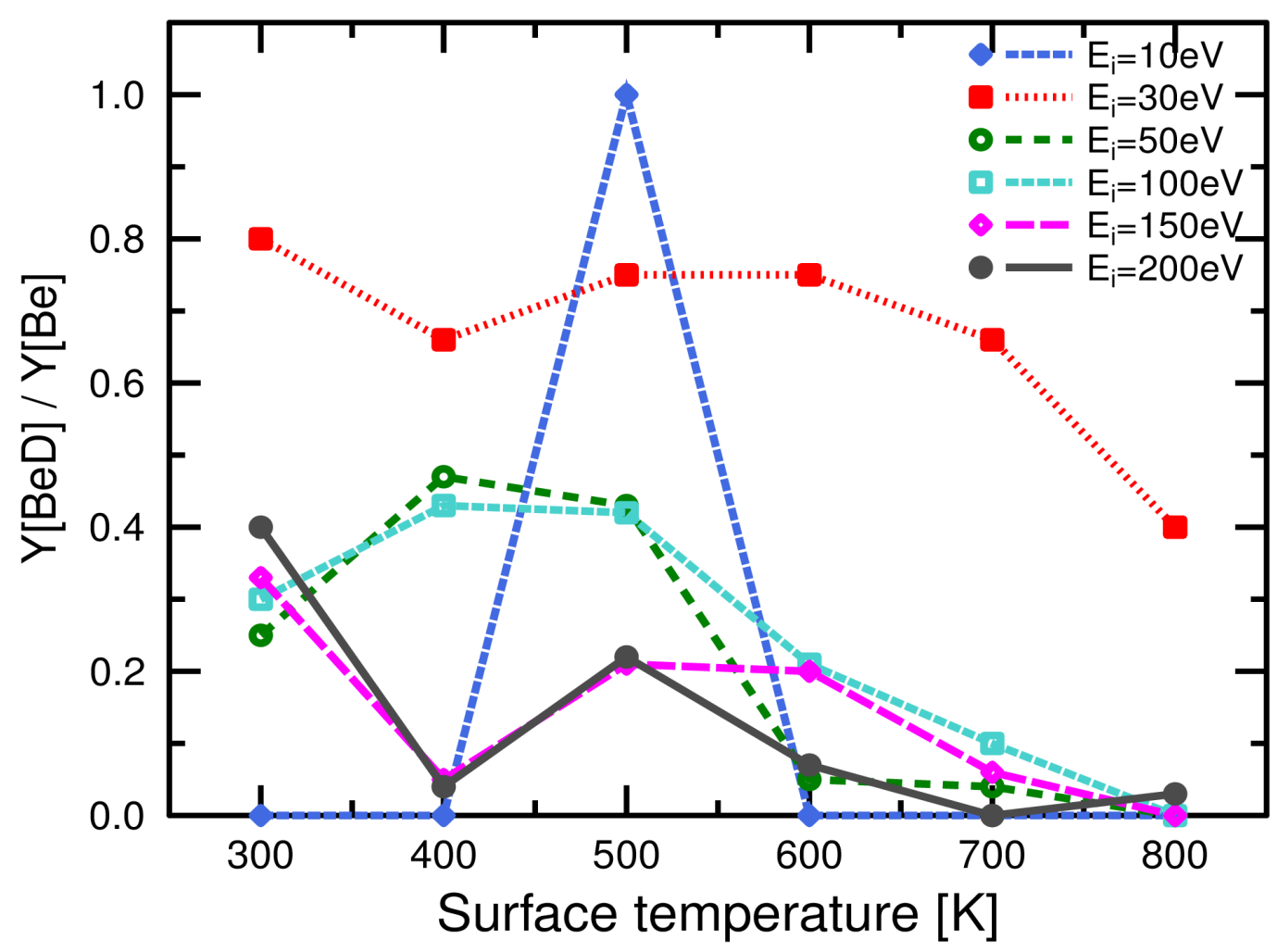

Fig. 5 The fraction of Be eroded as $\mathrm{BeD}$ molecules as a function of surface temperatures for different impact energies. 


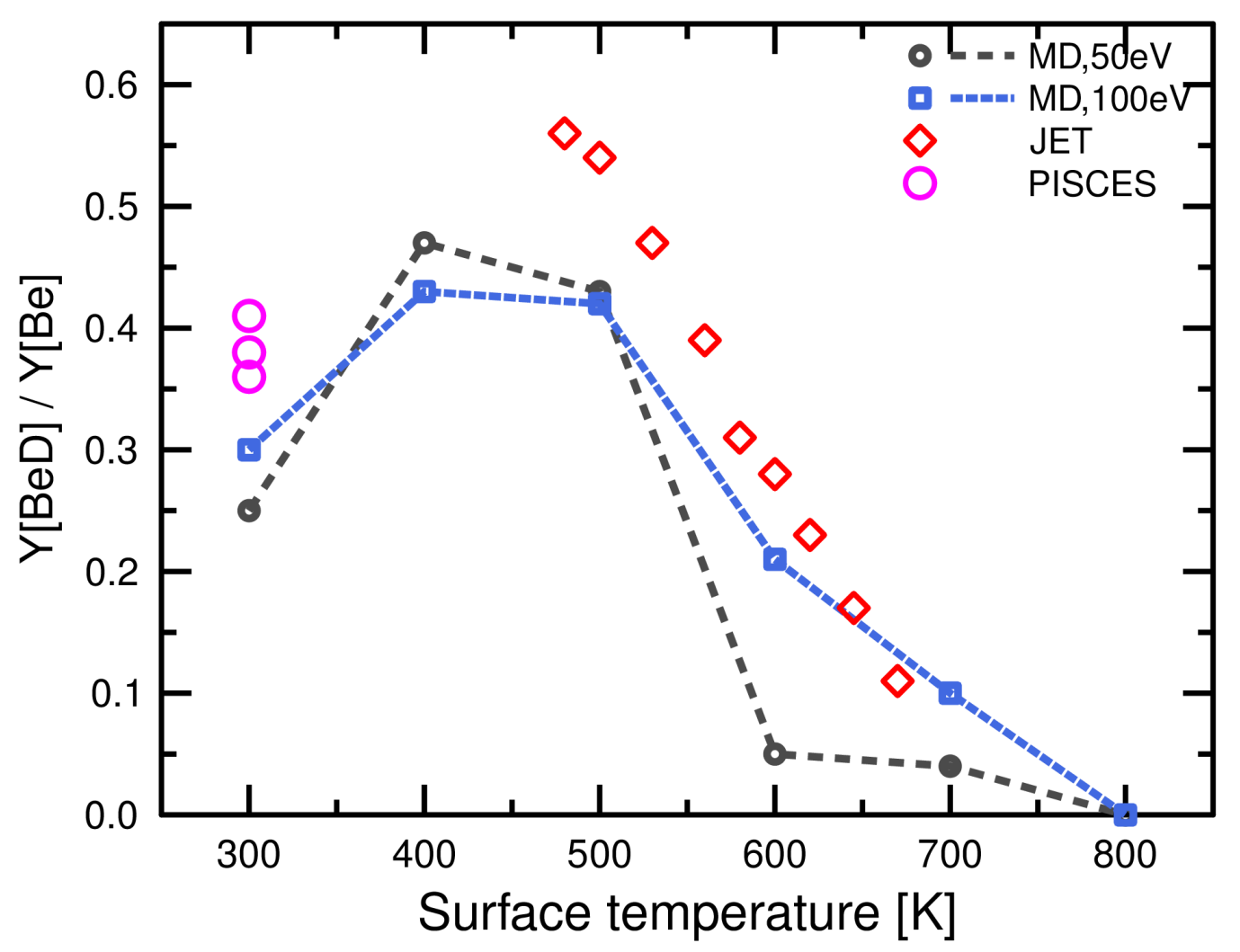

Fig. 6 A comparison of BeD:Be ratio obtained by MD and experiments done at JET and PISCES as afunction of surface temperatures. 


\section{References}

[1] J. Doggett, et al. , ITER Tokamak Device, International Atomic Energy Agency, Vienna, 1991.

[2] ITER Physics Basis Editors, ITER Physics Expert Group Chairs, Co-Chairs, ITER Joint Central Team, and Physics Integration Unit. Iter physics basis, Nuclear Fusion 39 (1999) 2137-2638.

[3] M. Merola, F. Escourbiac, A. Rene Raffray, ph. Chappuis, T. Hirai, s. Gicquel. Fusion Eng. Des. 96-97 (2015) 34-41

[4] G. Federici 2006 Plasma wall interactions in ITER Phys. Scr. T 124 1-8

[5] S. Brezinsek, M.F. Stamp, D. Nishijima, D. Borodin, S. Devaux, K. Krieger, S. Marsen, M. O'Mullane, C. Bjoerkas, A. Kirschner and JET EFDA contributors. NF. 54 (2014) 103001

[6] G. Duxbury, M.F. Stamp, H. Summers, Plasma Phys. Controlled Fusion 40 (1998) 361.

[7] R.P. Doerner, A. Grossman, S. Luckhardt, R. Seraydarian, F.c. Sze, D.g. Whyte, R.w. Conn, J. Nucl. Mater. 257 (1) (1998) 51-58.

[8] R.P. Doerner, M.J. Baldwin, D. Buchenauer, G. De Temmerman, D. Nishijima, J. Nucl. Mater. 390-391 (2009) 681-684.

[9] R.P. Doerner, C. Björkas, D. Nishijima, T. Schwarz-Selinger, J. Nucl. Mater. 438 (2013) 272275.

[10] J. Roth, W. Eckstein, M. Guseva, Fusion Eng. Des. 37 (1997) 465-480.

[11] A. Kirschner, V. Philipps, J. Winter, U. Kögler, Nucl. Fusion 40 (2000) 989

[12] C. Björkas, K. Nordlund, J. Nucl. Mater. 439 (2013) 174-179.

[13] C. Björkas, D. Borodin, A. Kirschner, R.K. Janev, D. Nishijima, R. Doerner, K. Nordlund, Plasma Phys. Controlled Fusion 55 (2013) 074004.

[14] C. Björkas, K. Vörtler, K. Nordlund, D. Nishijima, R. Doerner, New J. Phys. 11 (2009) 123017.

[15] A. Lasa, K. Schmid, K. Nordlund, Phys. Scr. T159 (2014) 014059.

[16] E. Safi, C. Björkas, A. Lasa, K. Nordlund, I. Sukuba, M. Probst. J. Nucl. Mat. 463 (2015) 805809

[17] Kristen A. Fichthorn and W. H. Weinberg. J. Chem. Phys. 95, (1991) 1090

[18] M. Jaraiz, Predictive Simulation of Semiconductor Processing, Springer-Verlag, Berlin, pp. 73-109.

[19] C. Domain, C. Becquart, L. Malerba. J. Nuc. Mat. 335 (2004) 121-145. 
[20] M. Caturla, N. Soneda, E. Alonso, B. Wirth, T. D. de la Rubia, J. Perlado, J. Nuc. Mat. 276 (2000) 13-21.

[21] A. B. Bortz, M. H. Kalos, J. L. Lebowitz, J. Computational Physics 17 (1975) 10-18.

[22] A. Voter, in: K. Sickafus, E. Kotomin, B. Uberuaga (Eds.), Radiation Effects in Solids, vol. 235 (2007), pp. 1-23.

[23] D. P. Landau, K. Binder, A Guide to Monte Carlo Simulations in Statistical Physics, Cambridge University Press, 2000.

[24] I. Martin-Bragado, A. Rivera, G. Valles, J. Gomez-Selles, M. Caturla. Comput. Phys. Commun. 184 (2013) 2703-2710.

[25] G. Valles, I. Martin-Bragado, K. Nordlund, A. Lasa, C. Bjorkas, E. Safi, J. M. Perlado, A. Rivera. J. Nucl. Mat. (2016), submitted for publication.

[26] S.C. Middleburgh, R.W. Grimes. Acta materiala 59, 18 (2011) 7095-7103.

[27] M. G. Ganchenkova, V. A. Borodin, Phys. Rev. B 75, 054108 (2007).

[28] A. Allouche, M. Oberkofler, M. Reinelt and Ch. Linsmeier, J. Phys. Chem. C, 114 (8), (2010) 3588-3598.

[29] M. G. Ganchenkova, V. A. Borodin, R. M. Nieminen, Phys. Rev. B 79, 134101 (2009).

[30] G. Henkelman, B. P. Uberuaga, H. Jónsson. J. Chem. Phys. 113, 9901 (2000).

[31] G. Kresse, J. Hafner. Phys. Rev. B 47, 558(R) (1993)

[32] P. Zhang, J. Zhao, B. Wen. J Phys Condens Matter. (2012) 24(9):095004.

[33] M.G. Ganchenkova, P.V. Vladimirov, V.A. Borodin. Fusion Reactor Mat. 386-388, (2009), 7981

[34] Rion A Causey, Hydrogen isotope retention and recycling in fusion reactor plasma-facing components, Vol. 300, Issues 2-3 (2002) 91-117

[35] H. Jonsson, G. Mills, K. W. Jacobsen, in Classical and Quantum Dynamics in Condensed Phase Simulations, B. J. Berne, G. Ciccotti, and D. F. Coker (Eds.) (World Scientific, 1998), p. 385.

[36] G. Henkelman, H. Jonsson, J. Chem. Phys. 113, 9978 (2000).

[37] J. F. Ziegler, M.D. Ziegler, J.P. Biersack. Nucl. Instr. and Meth. Phys. Research Sec. B. 268, 11-12 (2010), 1818-1823

[38] K. Nordlund, PARCAS computer code, (2006).

[39] C. Björkas, N. Juslin, H. Timko, K. Vörtler, K. Henriksson, K. Nordlund, P. Erhart, J. Phys. 
Condens. Matter 21 (2009) 445002.

[40] K. Vörtler, K. Nordlund, J. Phys. Chem. C 114 (2010) 5382-5390.

[41] D. Nishijima, R. P. Doerner, M. J. Baldwin, G. Temmerman. J. Nucl. Mater. (2009) 390-391 $132-5$

[42] R. Behrisch, W. Eckstein, Wolfgang (Eds.). Sputtering by Particle Bombardment: Experiments and Computer Calculations from Threshold to Mev Energies (2007) 